\title{
Novel Genetic Associations Between Lung Cancer and Indoor Radon Exposure
}

Jung Ran Choi ${ }^{1}$, Sang-Baek Koh ${ }^{1,2}$, Seong Yong Park ${ }^{3}$, Hye Ryun Kim ${ }^{4}$, Hyojin Lee ${ }^{5}$, Dae Ryong Kang ${ }^{6}$

${ }^{1}$ Institute of Genomic Cohort and ${ }^{2}$ Department of Preventive Medicine, Yonsei University Wonju College of Medicine, Wonju, ${ }^{3}$ Department of Thoracic and Cardiovascular Surgery, Yonsei University College of Medicine, ${ }^{4}$ Department of Internal Medicine, Yonsei Cancer Center, Yonsei University College of Medicine, ${ }^{5}$ Macrogen Inc., Seoul, ${ }^{6}$ Center of Biomedical Data Science, Yonsei University Wonju College of Medicine, Wonju, Korea

J Cancer Prev 2017;22:234-240

https://doi.org/10.15430/JCP.2017.22.4.234

The original version of this article contained error in the name of the fourth author which was given incorrectly as Hye Run Kim. The author's name should have been written as Hye Ryun Kim.

The authors would like to apologize for this mistake and any inconvenience it may have caused. 